\title{
Spontaneous Raman Scattering in Hollow Core Photonic Crystal Fibres
}

\author{
N.V. Wheeler ${ }^{1}$, M. G. Pappa ${ }^{1}$, T. D. Bradley ${ }^{1}$, Y. Chen ${ }^{1}$, W. Brooks ${ }^{2}$, J. Storey ${ }^{2}$, M. Foster ${ }^{2}$, D. J. Richardson ${ }^{1}$ and \\ M. N. Petrovich ${ }^{1}$ \\ ${ }^{1}$ Optoelectronics Research Centre \\ University of Southampton \\ Southampton, United Kingdom, SO17 1BJ \\ n.v.wheeler@soton.ac.uk \\ 2 IS Instruments Ltd \\ Pipers Business Centre \\ 220 Vale Rd, Tonbridge, Kent, United Kingdom, TN9 1SP \\ mfoster@is-instruments.com
}

\begin{abstract}
We report the first measurements of spontaneous Raman scattering from a gas sample confined in a Kagome lattice hollow core photonic crystal fibre. We compare the signal strength recorded in this fibre, with that from a similar length of hollow core photonic bandgap fibre and find that, even though the loss of the Kagome fibre is lower, the signal strength in the hollow core photonic bandgap fibre is significantly stronger. We speculate that this may be due to the different effective capture efficiencies of the two fibre types, which are defined by the fibres' numerical apertures.
\end{abstract}

Keywords-Microstructured fibres; Gas sensing; Spontaneous Raman Scattering; Hollow core photonic crystal fibres.

\section{INTRODUCTION}

Spontaneous Raman scattering is an attractive technique for optical gas sensing as the spectrum of the Stokes light provides a distinct signature or 'fingerprint' which is reflective of the gas composition. Multiple gas species can be quantitatively detected from a single-shot measurement, without a requirement for fine tuning the source to a specific gas absorption band, as typical of absorption based gas spectroscopy. Furthermore, Raman sensing is relatively insensitive to water vapour, which is a major drawback and limitation for conventional spectroscopy, where absorption from atmospheric water vapour is dominant in large regions of the spectrum in the near and the mid-infrared. However, the Raman signal is extremely weak which makes it extremely challenging to achieve the signal to noise ratio required for most real-world applications.

Hollow core photonic crystal fibres (HC-PCFs) [1] have recently been used to overcome this challenge [2-6]. In a HC$\mathrm{PCF}$, light is guided with low attenuation in an air core. This core can be filled with a gas sample to create a compact gas cell; in the context of Raman gas sensing, this cell provides an unprecedented interaction length between the confined gas species and the pump light, which is guided in the fibre core, while efficiently collecting and guiding the Stokes signal. Using this format, detection of both forward and backward Stokes

We acknowledge funding support from Innovate UK and the Royal Society. signals has been demonstrated. Additionally, sensitivities as low as $0.2 \mathrm{ppm}$ of methane have been achieved [6].

Up until now, all demonstrations of spontaneous Raman scattering in HC-PCFs have used hollow core photonic bandgap fibres (HC-PBGFs). In recent years, alternative types of $\mathrm{HC}$ PCFs have emerged, known as hollow core antiresonant fibres (HC-ARFs) or hollow core negative curvature fibres. These are distinct from HC-PBGFs as they do not rely on the photonic bandgap effect to guide light in an air core. Instead, light is trapped in specific spectral windows through anti-resonance with the core boundary. There have recently been many advances in the fabrication of these fibres which have stemmed from an improved understanding of how the core boundary shape affects the loss; the record low loss for a HC-ARF is now $7.7 \mathrm{~dB} / \mathrm{km} @ \sim 750 \mathrm{~nm}$, albeit with high bending loss, [7] and HC-ARFs can now achieve lower attenuation than HC-PBGFs at wavelengths below $\sim 1 \mu \mathrm{m}$. HC-ARFs are therefore an interesting prospect for application in Raman analysis of gas samples as the Raman signal is proportional to $\lambda^{-4}$, where $\lambda$ is the wavelength of the pump laser, and therefore operation at shorter wavelengths leads to a comparatively much higher signal strength.

In this paper, we report the first measurements of spontaneous Raman scattering from ambient air (detecting oxygen and nitrogen) in Kagome hollow core fibres (K-HCFs) which are a type of HC-ARF. Improvements in the attenuation of K-ARFs at a range of wavelengths have recently been reported [8]. The Stokes signal strengths recorded using the K$\mathrm{ARF}$ are compared to results from a similar length HC-PBGF, operating in the same conditions in the same spectral region. We find that, even with the lower attenuation offered by the K-HCF, the Stokes signal is $\sim 8$ times stronger in the HC-PBGF. We speculate that this may be due to the higher capture efficiency of the Stokes signal in a HC-PBGF, due to the higher numerical aperture (NA) of this fibre type; this has implications for optimisation of HC-PCF design for Raman gas sensing applications. 


\section{Key Properties of Hollow CORE PhOtONIC CRYSTAL FIBRES}

The power, $P_{S}$, of the spontaneous Raman Stokes signal can be expressed as [2],

$$
P_{S}=\sigma D P_{P} \Omega L,
$$

where $\Omega$ is the detected solid angle of the Stokes signal, $\sigma$ is the Raman scattering cross-section, $D$ is the gas density, $P_{P}$ is the incident pump laser power and $L$ is the interaction length. In the present work, we compare the performance of two types of HCPCF and from (1), any difference in detected signal for a given coupled laser power, for the same gas sample and same fibre length, can be attributed to the effective entendue of the fibre, given by the product $\Omega L$. The effective interaction length, $L$, is dependent on the fibre loss at the pump and Stokes wavelengths. In [2], $\Omega$ is approximated using the numerical aperture (NA) of the HC-PCF. For the experiments reported here, the pump laser wavelength is $786.4 \mathrm{~nm}$, and thus the Stokes signals of oxygen and nitrogen are expected to be at $896.0 \mathrm{~nm}$ and $962.9 \mathrm{~nm}$ [9] respectively. Thus, the two fibres which we test operate between $\sim 750 \mathrm{~nm}$ and $1 \mu \mathrm{m}$. The key properties of these fibres are described in the following sections.

\section{A. Hollow Core Photonic Bandgap Fibre}

The loss spectrum, measured using the cutback technique ( $284 \mathrm{~m}$ to $10 \mathrm{~m}$ ) for the HC-PBGF used in our experiments is shown in Fig. 1(a). The loss at the pump laser wavelength is $170 \mathrm{~dB} / \mathrm{km}$ and the loss at the oxygen and nitrogen Stokes wavelengths is $\sim 110 \mathrm{~dB} / \mathrm{km}$ and $500 \mathrm{~dB} / \mathrm{km}$ respectively (note that the loss at the nitrogen Stokes wavelength is estimated using measurements on shorter fibres due to the higher attenuation). In most cases, HC-PBGFs transmit light with low attenuation over a single spectral window, and within this spectral window, the low loss bandwidth is punctuated by 'surface modes'; these modes are localised in or around the silica core surround and at specific wavelengths they can couple with the low loss core guided modes to significantly increase attenuation. This is evident in Fig. 1(a) at wavelengths around $870 \mathrm{~nm}$ and $960 \mathrm{~nm}$ (the latter, notably in line with the nitrogen Stokes signal). An optical microscope image of the HC-PBGF is shown inset to Fig. 1 (a); the fibre has a 19-cell core and the core diameter is $\sim 16 \mu \mathrm{m}$.

\section{B. Kagome Hollow Core Fibre}

The loss spectrum for the K-HCF is shown in Fig. 1(b). This measurement was taken using the cutback technique (34 $\mathrm{m}$ to $5 \mathrm{~m})$. The loss at the pump laser wavelength is $\sim 60 \mathrm{~dB} / \mathrm{km}$ and the loss at the oxygen and nitrogen Stokes wavelengths is $\sim 100$ $\mathrm{dB} / \mathrm{km}$ and $\sim 170 \mathrm{~dB} / \mathrm{km}$ respectively. These losses are notably lower than for the HC-PBGF which should enable use of longer fibre lengths and lead to potentially higher Stokes signals. Although it should be noted here that K-HCFs have much higher bending losses than HC-PBGFs. Additionally, no surface mode resonances are present within this transmission window and a broader spectral bandwidth is present which is amenable for detecting a wider range gas species with more widely varying Raman shifts. Furthermore, the overlap of the core guided light with the silica fibre cladding in a K-ARF is at least an order of magnitude lower than in a HC-PBGF and this should act to reduce the Raman signal which is collected from the silica cladding; this silica Raman signal has been previously noted to

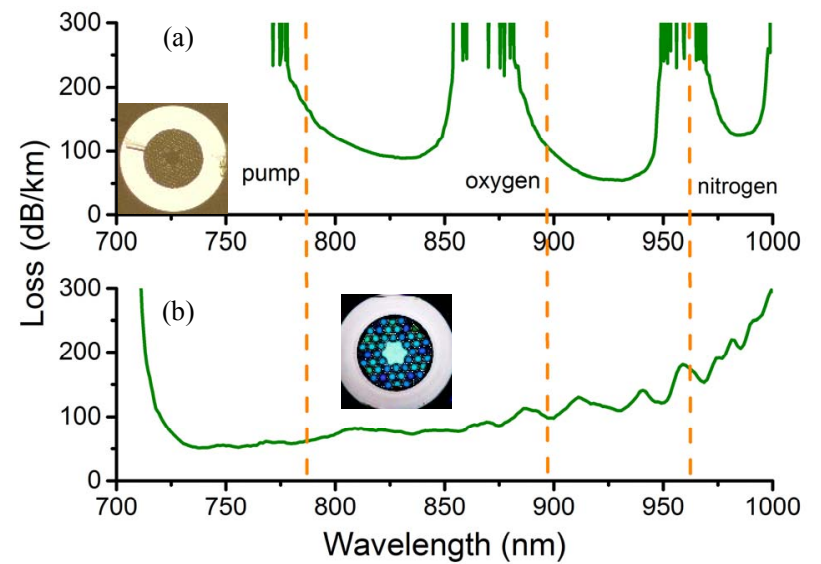

Fig. 1(a): Attenuation of the HC-PBGF used in experiments as measured using the cutback technique, on a length of HC-PBGF cutback from 284 $\mathrm{m}$ to $10 \mathrm{~m}$. (b) Attenuation of the K-HCF used in experiments as measured using the cutback technique, on a length of K-HCF cutback from $34 \mathrm{~m}$ to $5 \mathrm{~m}$.

be an issue in achieving high signal to noise in previous publications [e.g., 2]. An optical microscope image of the KHCF is shown inset to Fig. 1(b); the fibre has a 7-cell core and the core diameter is $\sim 54 \mu \mathrm{m}$, which is significantly larger than the PBGF and this can be advantageous for fast loading of gas samples into the fibre.

\section{EXPERIMENTAL SET-UP}

Light from a single mode laser diode $(786.4 \mathrm{~nm})$ is launched into the HC-PCF sample via a telescope lens arrangement. The lens choice is tailored for each experiment to maximise the light coupled into the fundamental mode of the HC-PCF under test. For both the HC-PBGF and the K-HCF, the fibre length was $2.7 \mathrm{~m}$ and the gas sample was ambient air. At the fibre output, pump light is removed using a long wavelength bandpass filter with a cut-off at $\sim 820 \mathrm{~nm}$ and the forward-scattered Stokes signal is coupled to a spectrometer via a multimode patch-cord. The spectrometer is a custom designed (IS-Instruments) dispersive spectrometer in a Czerny-Turner configuration which directs the input light onto a peltier-cooled Andor iVac324 with a 200x1650 pixel array. The spectral response of the spectrometer was calibrated using a Xenon lamp. The wavelength-dependent response of the signal strength collected by the spectrometer is calibrated (for each measurement trace) using a flat-field correction. Note that, using the lens launch arrangement and the pump laser, the fibre attenuation at the pump wavelength was checked by a further loss measurement; for the K-HCF the pump loss was measured to be $\sim 30 \mathrm{~dB} / \mathrm{km}$. This lower value may be due to positioning of the fibre (as it is sensitive to bend loss) and/or improved coupling to the fundamental mode with the lens launch.

\section{RESULTS AND DISCUSSION}

A first comparison of the Raman Stokes signals from $2.7 \mathrm{~m}$ of HC-PBGF and K-HCF is shown in Fig. 2, for a coupled pump power of $\sim 50 \mathrm{~mW}$ and an integration time of $60 \mathrm{~s}$. Two key differences between the two fibres are immediately evident from these traces. Firstly, the Raman Stokes signal from the HCPBGF is significantly stronger; this is especially surprising for 


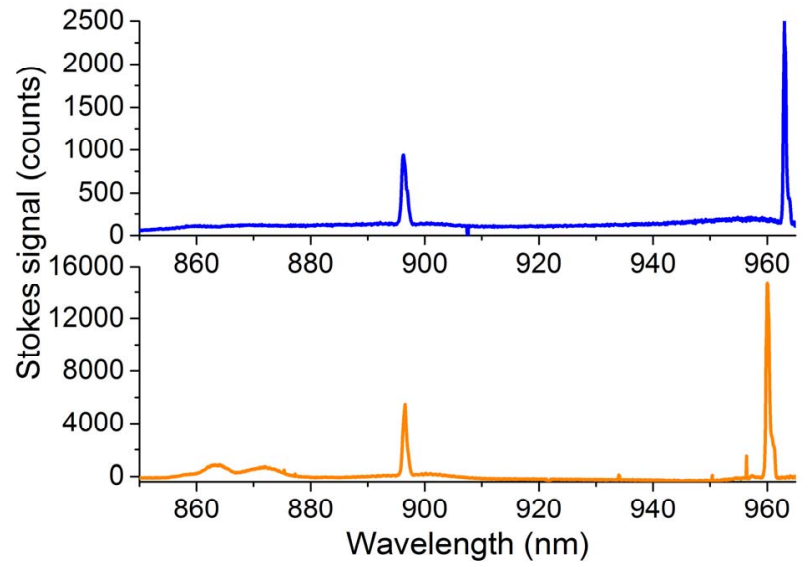

Fig. 2: Experimental traces recorded using $2.7 \mathrm{~m}$ of K-HCF (top) and 2.7 $\mathrm{m}$ of HC-PBGF (bottom) for a coupled laser power of $\sim 50 \mathrm{~mW}$. Two peaks are evident (oxygen Stokes signal at $\sim 896 \mathrm{~nm}$ and nitrogen Stokes at $\sim 962 \mathrm{~nm}$ ). Silica Raman signal is evident between 860 and $880 \mathrm{~nm}$ in the HC-PBGF.

the nitrogen Stokes signal, as in the HC-PBGF both the pump laser light and the nitrogen Stokes light experience much higher attenuation than in the K-HCF (if we assume that the Stokes light is captured by the fundamental mode of the fibres). Secondly, the signal from the silica Raman is much weaker, as expected, in the K-HCF.

The evolution of the Raman Stokes signals of both oxygen and nitrogen were characterised further in both fibres as a function of laser power. The sum of the Stokes signal for each gas in each fibre is plotted as a function of laser output power and coupled laser power in Fig. 3(a) and (b) respectively. The coupled laser power represents the estimated power coupled into the core of the HC-PCF under test and is estimated using the fibre loss at the pump wavelength. The coupling efficiency of pump light was $\sim 50 \%$ into the HC-PBGF and $\sim 90 \%$ into the KHCF and even with significantly lower coupling efficiency, Fig. 3(a) shows that the HC-PBGF still outperforms the K-HCF in terms of signal strength.

A linear fit was applied to each set of data shown in Fig. 3(b). From (1), the slope of this fit represents the product, $\sigma D \Omega L$. Therefore, if we first consider the nitrogen Stokes lines data from the HC-PBGF and the K-HCF, and calculate the ratio of the linear fits in this case, we can calculate the ratio of the entendues of the two different fibres. From this calculation we find that the entendue of the HC-PBGF is $\sim 8$ times larger than the K-HCF for the nitrogen data. Similar analysis for the oxygen data implies the entendue of the HC-PBGF is $\sim 7$ times larger. As for both the pump and Stokes lines, the K-HCF attenuation is lower, this suggests that the significant factor here is the NA of each fibre. This is corroborated by the increase in entendue at longer wavelengths (even with a larger loss discrepancy), as the NA of a HC-PBGF increases with wavelength in the low loss spectral window [10].

The aim of this work was to compare the performance of KHCFs and HC-PBGFs for Raman gas sensing. From these early results, we can see that while the background signal due to silica Raman is significantly reduced in K-HCF, the Stokes signals from the gas species confined in the fibre core are also
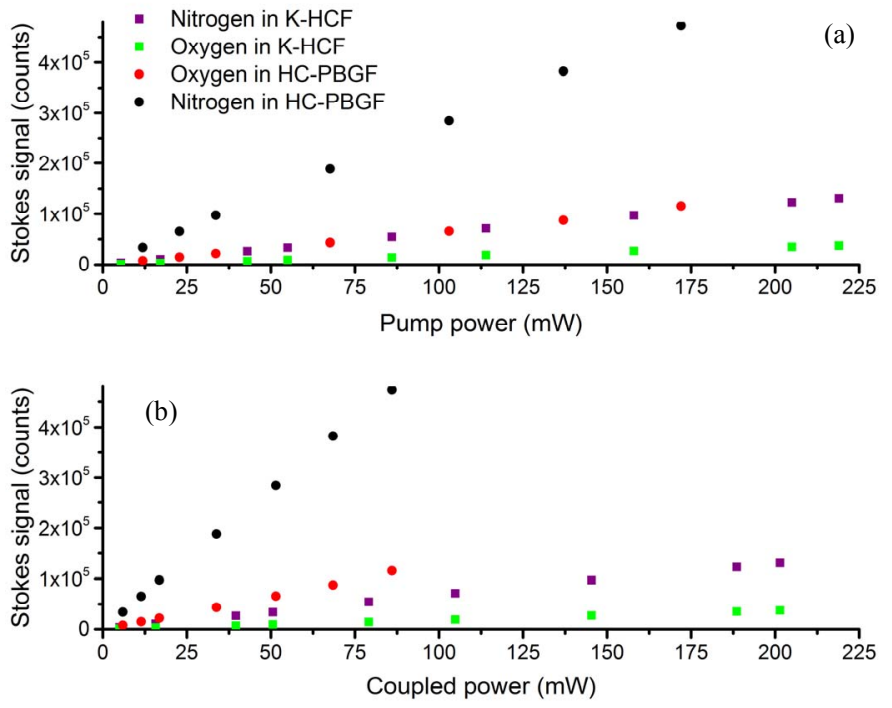

Fig. 3(a) and (b): Evolution of Raman Stokes signals in HC-PBGF and KHCF with pump output power and coupled pump power respectively.

significantly lower. We speculate that this is due to a lower capture efficiency in K-HCF compared to HC-PBGF and further work is underway to confirm this, the results of which will influence the design of the optimum HC-PCF for this application. The effect of fibre length is also being investigated in order to determine what conditions, in terms of fibre attenuation and fibre length, could enable a K-HCF to compete with a HC-PBGF, in order to be able to take advantage of the lower silica noise and wide bandwidth offered by this fibre design.

\section{REFERENCES}

[1] F.Poletti, M.N.Petrovich and D.J. Richardson, "Hollow-core photonic bandgap fibers: technology and applications," Nanophotonics 2 (5-6) 315 (2013).

[2] M. P. Buric, K. P. Chen, J. Falk, and S. D. Woodruff, "Enhanced spontaneous Raman scattering and gas composition analysis using a photonic crystal fiber," Applied Optics 47(23) 4255 (2008).

[3] K. K. Chow, M. Short, S. Lam, A. McWilliams, and H. Zeng, "A Raman cell based on hollow core photonic crystal fiber for human breath analysis," Medical Physics 41, 092701 (2014).

[4] S. Hanf, T. Bögözi, R. Keiner, T. Frosch, and J. Popp, "Fast and Highly Sensitive Fiber-Enhanced Raman Spectroscopic Monitoring of Molecular $\mathrm{H} 2$ and CH4 for Point-of-Care Diagnosis of Malabsorption Disorders in Exhaled Human Breath," Analytical Chemistry 87982 (2015)

[5] M. P. Buric, K. P. Chen, J. Falk, and S. D. Woodruff, "Improved sensitivity gas detection by spontaneous Raman scattering," Applied Optics 48(22) 4424 (2009).

[6] S. Hanf, R. Keiner, D. Yan, J. Popp, and T. Frosch, "Fiber-Enhanced Raman Multigas Spectroscopy: A Versatile Tool for Environmental Gas Sensing and Breath Analysis," Analytical Chemistry 865278

[7] B. Debord, A. Amsanpally, M. Chafer, A. Bas, M. Maurel, J-M. Blondy, E. Huggonot, L. Vincetti, , F. Gerome and F. Benabid, Optica, 4(2), 209 (2017).

[8] N.V. Wheeler et al., "Low Loss Kagome Hollow Core Fibers Operating from the Near- to the Mid-IR," Optics Letters (in press) (2017).

[9] W.R. Fenner, H. A. Hyatt, J. M. Kellam and S. P. S. Porto, "Raman cross section of some simple gases," J. Opt. Soc. Am. 63(1) (1973).

[10] M.J.F. Digonnet, H.K.Kim, G.S. Kino and S. Fan, "Understanding AirCore Photonic-Bandgap Fibers: Analogy to Conventional Fibers," 23(12) 4169 (2005). 